\title{
Kaons at CERN: The NA62 experimental program
}

\author{
Augusto Ceccucci ${ }^{* \dagger}$ \\ CERN, 1211 geneva 23 \\ E-mail: augusto.ceccuccidcern.ch
}

Quark mixing and CP-Violation has been a very active area of investigation over the past decades. Owing to the last round of experiments in $K$ and $B$ mesons, our understanding is now completely compatible with the existence of just one phase in the Cabibbo-Kobayashi-Maskawa mixing matrix. The precision of the tests in the quark sector can further improve thanks to the interplay of theory and experiments. Flavour transitions are so sensitive to short distance mechanisms that we are compelled to press for more quantitative tests of the Standard Model (SM) studying rare processes involving quarks and leptons. This is especially important because the direct exploration of the energy frontier is currently limited at the LHC energies and may remain so for many years to come. The paper presents the status of NA62, the new rare kaon decay at the CERN SPS

The 8th International Workshop on Chiral Dynamics, CD2015 ***

29 June 2015 - 03 July 2015

Pisa, Italy

* Speaker.

$\dagger$ 


\section{Introduction}

Quark mixing and CP-Violation has been a very active area of investigation over the past decades. Owing to the last round of experiments in $K$ and $B$ mesons, our understanding is now completely compatible with the existence of just one phase in the Cabibbo-Kobayashi-Maskawa mixing matrix $[1,2]$. The precision of the tests in the quark sector is improving thanks to the interplay of theory and experiments. Flavour transitions are so sensitive to short distance mechanisms that we need to press for quantitative tests of the Standard Model (SM). Since the directly accessible energy frontier is limited (LHC for the foreseeable future) it is important to continue to explore the zepto-Universe $\left(10^{-21} \mathrm{~m}\right)$ indirectly with rare processes. Among the reasons that make these studies compelling, it is very prominent the attempt to explain the Baryon Asymmetry of the Universe (BAU). This refers to the small but essential non zero value of baryons over photons $\left(\approx 5 \times 10^{-10}\right)$ in the current Universe. Matter and Antimatter should have been made in the same amounts in the Big Bang and should have completely disappeared due to mutual annihilation, that a tiny fraction of matter survived is one of the biggest unresolved puzzles of Nature. To explain $\mathrm{BAU}$, at least from a qualitative point of view, CP-Violation is a necessary ingredient together with baryon number violation and thermal non-equilibrium (Sakharov, [3]). CP-Violation has been observed in the mixing and decay of $K$ and $B$ mesons, it is classified in three different forms: direct, indirect, interference, but the bottom line is that all CP-Violating phenomena can at this moment be interpreted as manifestations of a single complex phase in the quark mixing. To stress the importance of flavour physics is sufficient to remind that the origin of the quark masses is related to the acquired vacuum expectation value (VEV) of the Higgs field, flavour and Higgs are intrinsically related by the Yukawa interaction of the Higgs condensate. Charged weak currents are responsible of the transitions among quarks. The properties of these transitions have very big implications. For instance, given $n$ families of quarks, the simple conservation of probability (unitarity of the transitions) leads to the cancelation of flavour changing neutral currents (FCNC) at tree level (Weinberg). As important, the quasi degenerate nature of the masses of the quarks, leads to a suppression of higher order FCNC, a mechanism known as GIM [4]. These are the suppressions that make FCNC such a sensitive test of SM: if measured to differ from the SM predictions it would give a clear sign of physics beyond the SM!

While the collider frontier is limited by the reach of the LHC in terms of energy in the proton proton center of mass, in principle no such limitation exists for rare decays. Rare decays might well turn out to remain the only road to open a window at high energy in the foreseeable future. Excellent reviews exist to grasp the status of the quark transitions and their implications (e.g. CPViolation and CKM reviews in [5] ). The aim of flavour physics is two-fold. Within the SM we can determine fundamental parameters like quark masses, mixing parameters and phenomenological quantities like decay constants and form factors allowing us to move from the realm of hadrons to the underlying world of the quarks, the fundamental constituents. On the other side, we can fix the SM parameters using theoretical and phenomenological determinations (lattice QCD, large- $\mathrm{N}$ phenomenology, chiral perturbation theory etc.) to make firm predictions and look for deviations in the data. This second approach works particularly well for the kaons because the lattice QCD and chiral perturbation theory are excellent tools in this domain. 


\begin{tabular}{|c|c|c|}
\hline Decay & \multicolumn{2}{|c|}{ Branching Ratio $\left(\times 10^{-10}\right)$} \\
\hline & Theory & Experiment \\
\hline$K^{+} \rightarrow \pi^{+} v \bar{v}$ & $0.911 \pm 0.072[6]$ & $1.75_{-1.05}^{+1.15}[7]$ \\
$K_{\mathrm{L}}^{0} \rightarrow \pi^{0} v \bar{v}$ & $0.30 \pm 0.03[6]$ & $<260($ CL 90\%) [8] \\
\hline
\end{tabular}

Table 1: Important rare kaon decays

\section{Rare $K$ Decays}

As mentioned earlier, what sets rare kaon decays apart from the rest is that the theoretical predictions are particularly robust. In addition, the experimentation does not depend on the availability of the highest possible centre of mass energy so that the onus is purely on the experimenter side, where ingenuity can still lead to breakthroughs without having to wait for someone else to build a new accelerator (essentially with a time scale outside the event horizon for most of us). The most interesting rare kaon decays are presented in Table 1, where the experimental status of the art is compared with the theoretical predictions. The gap between theory and experiment is striking. This window of opportunity is addressed by the CERN NA62 experiment $[9,10]$ for the charged mode and by the KOTO Experiment [11] for the neutral mode. The rest of the article presents the status of NA62.

\section{Status of NA62}

It is a unique combination of the availability of high energy protons from the CERN Super Proton Synchrotron (SPS) and the significant expertise available at CERN in the study of kaons that made possible to envisage and propose an experiment able to study the elusive $K^{+} \rightarrow \pi^{+} v \bar{v}$ with a novel in-flight technique. To do so, however, required the development of new detector technologies, in particular the development of a high rate beam silicon pixel tracker (hybrid) with 200 ps time resolution per hit (Gigatracker or GTK) [12]. The three GTK stations sit in hostile beam conditions and to preserve their lifetime they are cooled to $-20^{\circ} \mathrm{C}$ by an innovative microchannel system. Sensor (200 $\mu \mathrm{m} \mathrm{Si})$, TDCpix ASIC (100 $\mu \mathrm{m}$ thick) and microchannel plates together contribute to less than $0.5 \%$ of a radiation length per station. A picture of the NA62 Gigatracker is shown in Fig. 1 A picture of the NA62 apparatus at CERN is shown in Fig. 2. Crucial detectors also include a large size straw tracker operated in the decay tank, liquid krypton electromagnetic calorimetry, full coverage of photon veto detectors and Ring Imaging Cherenkov counter (RICH) for $\pi / \mu$ separation at high momentum. NA62 has started data taking in October 2014. The main kinematic features of kaon decays can be appreciated in the data reconstructed using the magnetic spectrometer (straw) (Fig. 3). To give an idea about the quality of the liquid krypton calorimeter $\pi^{0}$ reconstruction, one can appreciate in Fig. 4 the $\pi^{+}$squared reconstructed mass resolution for $K^{+} \rightarrow \pi^{+} \pi^{0}, K^{+} \rightarrow \pi^{+} \pi^{0} \pi^{0}$ and the $v$ squared reconstructed mass for $K^{+} \rightarrow \pi^{0} e^{+} v$ respectively. NA62 will continue data taking at least until the CERN Long Shutdown 2 (LS2) at the end of 2018. By then it should have collected a flux of about $10^{13} \mathrm{kaon}$ decays, enough to make a $10 \%$ determination of the $K^{+} \rightarrow \pi^{+} v \bar{v}$ branching ratio. The physics program of NA62 also include a rich menu of lepton flavour violation, lepton universality and chiral perturbation theory measurements. More details on the preliminary analyses can be found in the CERN EP seminar [13]. 


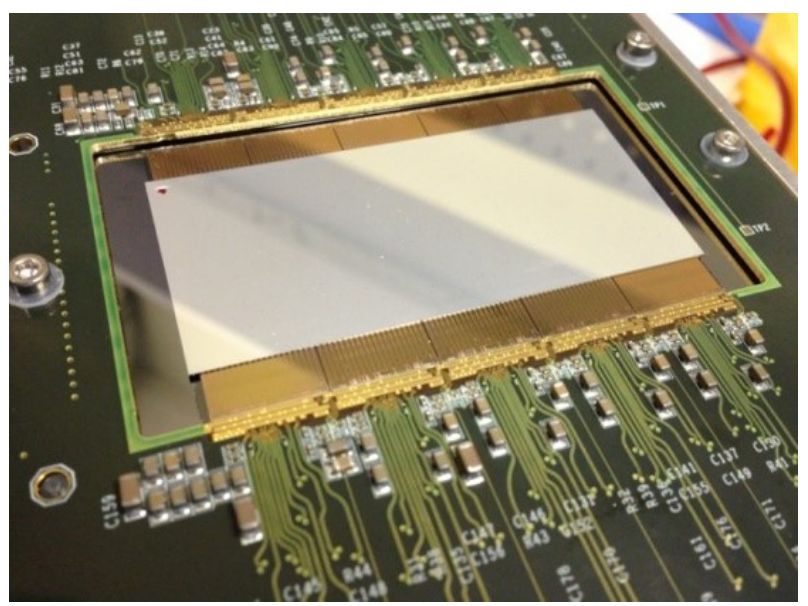

Figure 1: One station of the NA62 Gigatracker. The detector can track $10^{9}$ particles per second (hence the name!) with a pixel pitch of $300 \mu \mathrm{m}$ and a time resolution of $200 \mathrm{ps}$ per hit. One station is composed by 10 TDCPix ASICs for a total of $\approx 60 \times 30 \mathrm{~mm}^{2}$.

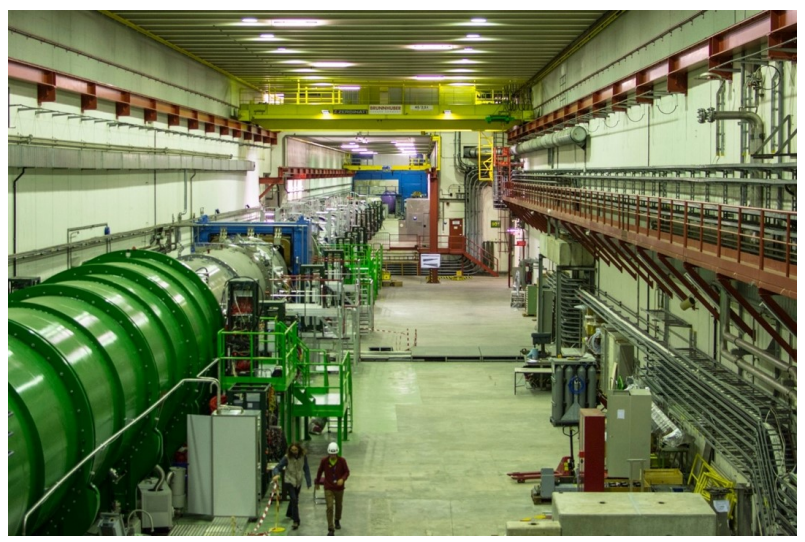

Figure 2: NA62 ready to take data

\section{References}

[1] N. Cabibbo, “Unitary Symmetry and Leptonic Decays,” Phys. Rev. Lett. 10 (1963) 531.

[2] M. Kobayashi and T. Maskawa, "CP Violation in the Renormalizable Theory of Weak Interaction," Prog. Theor. Phys. 49 (1973) 652.

[3] A. D. Sakharov, "Violation of CP Invariance, c Asymmetry, and Baryon Asymmetry of the Universe," Pisma Zh. Eksp. Teor. Fiz. 5 (1967) 32 [JETP Lett. 5 (1967) 24] [Sov. Phys. Usp. 34 (1991) 392] [Usp. Fiz. Nauk 161 (1991) 61].

[4] S. L. Glashow, J. Iliopoulos and L. Maiani, "Weak Interactions with Lepton-Hadron Symmetry," Phys. Rev. D 2 (1970) 1285.

[5] K. A. Olive et al. [Particle Data Group Collaboration], "Review of Particle Physics," Chin. Phys. C 38 (2014) 090001.

[6] A. J. Buras, D. Buttazzo, J. Girrbach-Noe and R. Knegjens, " $K^{+} \rightarrow \pi^{+} v \bar{v}$ and $K_{L} \rightarrow \pi^{0} v \bar{v}$ in the Standard Model: Status and Perspectives," arXiv:1503.02693 [hep-ph]. 


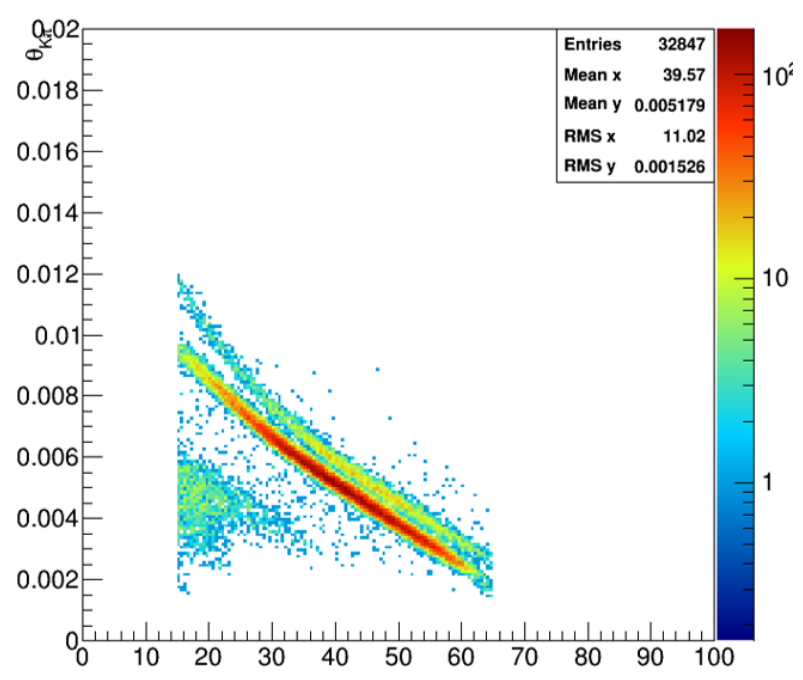

Figure 3: Distribution of the pion-kaon angle (rad) versus track momentum $(\mathrm{GeV} / \mathrm{c})$ based on the selection of one charged track in the straw magnetic spectrometer (NA62 preliminary)
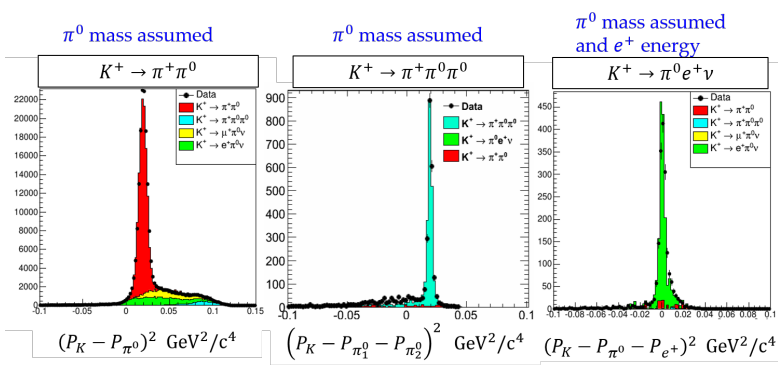

Figure 4: Squared reconstructed $\pi^{+}$and $v$ masses for kaon decays reconstructed using only liquid krypton calorimeter data (NA62 Preliminary)

[7] A. V. Artamonov et al. [ BNL-E949 Collaboration ], "Study of the decay $K^{+} \rightarrow \pi^{+} v \bar{v}$ in the momentum region $140 \leq \mathrm{P}(\pi) \leq 199 \mathrm{MeV} / \mathrm{c}$," Phys. Rev. D79 (2009) 092004. [arXiv:0903.0030 [hep-ex]].

[8] J. K. Ahn et al. [E391a Collaboration], Phys. Rev. D 81 (2010) 072004 [arXiv:0911.4789 [hep-ex]].

[9] G. Anelli et al., CERN-SPSC-2005-013, CERN-SPSC-P-326.

[10] F. Newson et al., "Prospects for $K^{+} \rightarrow \pi^{+} v \bar{v}$ at CERN in NA62," arXiv:1411.0109 [hep-ex].

[11] M. Togawa, J. Phys. Conf. Ser. 556 (2014) 1, 012046.

[12] B. Velghe et al., PoS Vertex 2014, 018 (2015).

[13] Giuseppe Ruggiero, "The NA62 Experiment: Status and Perspectives, CERN EP seminar, March 10, 2015, https://indico.cern.ch/event/360237/ 